\title{
Iterative Design of an Upper Limb Rehabilitation Game with Tangible Robots ${ }^{*}$
}

\author{
Arzu Guneysu Ozgur \\ EPFL, CHILI \\ Lausanne, Switzerland \\ arzu.guneysu@epfl.ch

\section{Kshitij Sharma} \\ NTNU \\ Trondheim, Norway \\ kshitij.sharma@ntnu.no \\ Francesco Mondada \\ EPFL, LSRO \\ Lausanne, Switzerland \\ francesco.mondada@epfl.ch
}

\author{
Maximilian Jonas Wessel \\ EPFL, UPHUMMEL \\ Center for Neuroprosthetics \\ Brain Mind Institute \\ Geneva, Switzerland \\ maximilian.wessel@epfl.ch \\ Ayberk Özgür \\ EPFL, CHILI \\ Lausanne, Switzerland \\ ayberk.ozgur@epfl.ch
}
Friedhelm Christoph Hummel EPFL, UPHUMMEL
Center for Neuroprosthetics
Brain Mind Institute
Clinique Romande de Réadaptation
Switzerland
friedhelm.hummel@epfl.ch

\author{
Wafa Johal \\ EPFL, CHILI, LSRO \\ Lausanne, Switzerland \\ wafa.johal@epfl.ch
}

\begin{abstract}
Rehabilitation aims to ameliorate deficits in motor control via intensive practice with the affected limb. Current strategies, such as one-on-one therapy done in rehabilitation centers, have limitations such as treatment frequency and intensity, cost and requirement of mobility. Thus, a promising strategy is home-based therapy that includes task specific exercises. However, traditional rehabilitation tasks may frustrate the patient due to their repetitive nature and may result in lack of motivation and poor rehabilitation. In this article, we propose the design and verification of an effective upper extremity rehabilitation game with a tangible robotic platform named Cellulo as a novel solution to these issues. We first describe the process of determining the design rationales to tune speed, accuracy and challenge. Then we detail our iterative participatory design process and test sessions conducted with the help of stroke, brachial plexus and cerebral palsy patients (18 in total) and 7 therapists in 4 different therapy centers. We present the initial quantitative results, which support several aspects of our design rationales and conclude with our future study plans.
\end{abstract}

${ }^{*}$ Produces the permission block, and copyright information

Permission to make digital or hard copies of all or part of this work for personal or classroom use is granted without fee provided that copies are not made or distributed for profit or commercial advantage and that copies bear this notice and the full citation on the first page. Copyrights for components of this work owned by others than the author(s) must be honored. Abstracting with credit is permitted. To copy otherwise, or republish, to post on servers or to redistribute to lists, requires prior specific permission and/or a fee. Request permissions from permissions@acm.org.

HRI '18, March 5-8, 2018, Chicago, IL, USA

( 2018 Copyright held by the owner/author(s). Publication rights licensed to Association for Computing Machinery.

ACM ISBN 978-1-4503-4953-6/18/03 ..\$15.00

https://doi.org/10.1145/3171221.3171262

\section{CCS CONCEPTS}

- Human-centered computing $\rightarrow$ Usability testing; Field studies; Haptic devices; Participatory design; • Computer systems organization $\rightarrow$ Robotics; • Computing methodologies $\rightarrow$ Multi-agent systems; $\bullet$ Applied computing $\rightarrow$ Life and medical sciences;

\section{KEYWORDS}

Gamified Rehabilitation; Tangible Robots; Rehabilitation Robotics; Iterative Design; Human-Robot Interaction; Haptic Interfaces

\section{ACM Reference Format:}

Arzu Guneysu Ozgur, Maximilian Jonas Wessel, Wafa Johal, Kshitij Sharma, Ayberk Özgür, Philippe Vuadens, Francesco Mondada, Friedhelm Christoph Hummel, and Pierre Dillenbourg. 2018. Iterative Design of an Upper Limb Rehabilitation Game with Tangible Robots. In Proceedings of 2018 ACM/IEEE International Conference on Human-Robot Interaction, Chicago, IL, USA, March 5-8, 2018 (HRI '18), 10 pages.

https://doi.org/10.1145/3171221.3171262

\section{INTRODUCTION}

Upper arm rehabilitation mainly focuses on relearning of lost or weakened functional movements that are crucial for daily life activities. Effective recovery process includes large volumes of repetitive exercises that induce neural plasticity. This plasticity is mediated via various mechanisms, e.g. anatomical or synaptic plasticity or adaptation to cortical circuitry or brain networks [24], which allows the neurons to respond and compensate for injury and disease [22]. Several studies have found that this intensive therapy in both acute and chronic phases improves motor function in patients [12, 55, 58]. Therefore, the process takes time and after some progress in initial 


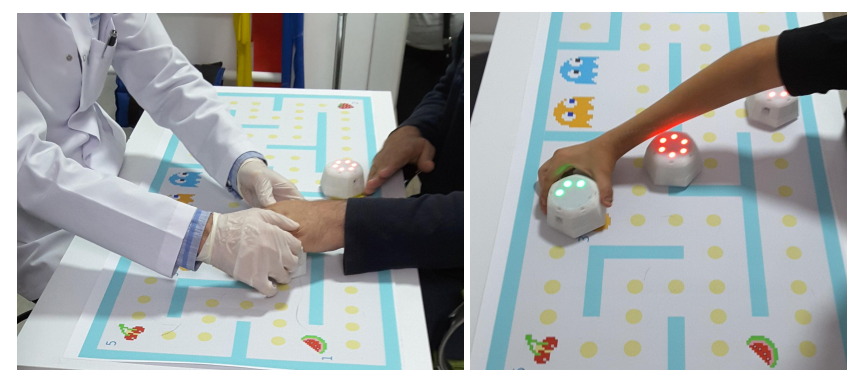

Figure 1: Gamified upper arm rehabilitation with tangible robots. Therapist can hold the patient's hand and easily assists the game play if needed.

therapy at the hospital and rehabilitation centers the patient continues with one to one basis therapy sessions. Due to the high cost and restricted availability of one to one therapy sessions, in the outpatient set-up, the goal of the long term treatment has to be that the patient can practice the exercises alone and unsupervised in the home-based environment. However, these intensive take-home exercises are criticized for being frustrating and boring. For example, daily exercise routines may include cleaning table surfaces with a towel. Besides, therapists cannot directly assess the quality of movements performed at home. Unfortunately, only 29 to $31 \%$ of patients carry out home-based exercise routines as recommended $[6,48]$. Some of the reasons behind the poor rehabilitation reported by the patients are as follows: Lack of time (28\%), forgetting to do the exercises (24\%), feeling that there was no improvement (10\%), exercises being too boring (25\%) and not remembering how to do the exercises him/herself at home (19\%) [6]. Therefore, transforming laborious repetitive exercises into enjoyable, motivating, easy to remember and intuitive experiences, and giving proper feedback about the performance of the user to him/herself as well as to the therapist are crucial factors for home therapy.

Ranging from assistive robotic systems to console based gaming activities, many interventions have been investigated by researchers to make this training more fun for patients. Recent studies show that technologies such as virtual environments, video capture systems, game consoles and robots can be a motivating and effective way of providing upper arm rehabilitation [5, 8, 9, 17, 18, 31, 36, 37, 47, 52]. Virtual environments with gamified exercises can provide customizable training and encourage the patient to become more motivated and involved in his/her rehabilitation [5, 36, 47]. Robotic arm aided rehabilitation is also shown to be effective in repetitive movement training $[17,18,31]$. Webcam-based video capture systems and game consoles such as the Nintendo Wii and Playstation II EyeToy deliver relatively cheap low cost solutions compared to relatively high cost robotic systems [8].

However, these systems generally need a special room or an isolated part in the therapy center and home. Their need of a computer or a TV makes the system difficult to be carried to different places and be set up. Taking into account the present significant challenges of the design of at home rehabilitation technologies such as the repurposing of use of rooms or shared devices such as TV and computer and space available in the home, above mentioned systems may not provide a proper solution $[4,19]$.

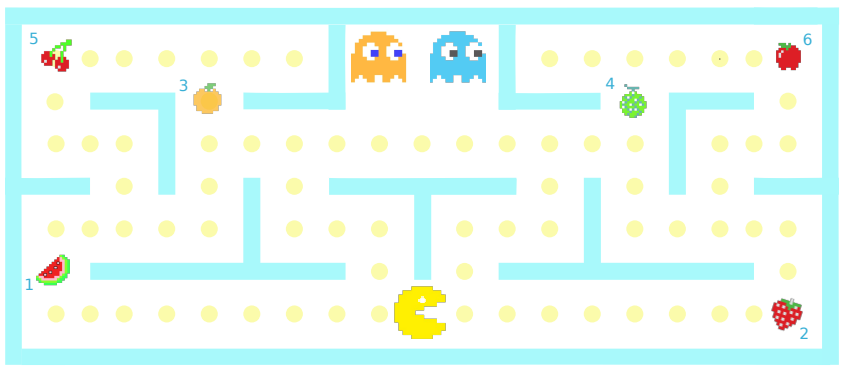

Figure 2: The patterned paper with the maze and visual game elements.

Current conventional physical and occupational therapy methods involve the manipulation of real objects of daily life and these therapy settings require a tangible setup where the user grasps, holds and moves objects [56,59]. To provide more compact and intuitive rehabilitation for home-therapy recent studies combine low-cost materials with small interactive tangible devices.

Studies show that utilizing tangible objects that the elderly are familiar with and providing a simple tangible interface with simplified elements may reduce the learning curve among elderly [3, 27, 57]. Tangible user interfaces also support post-stroke training through the integration of physical representations with digital representations by allowing the tracking of the training performance and progress of the patient objectively $[53,57]$.

Inspired by these promising results in tangibles for rehabilitation, we propose the use of Cellulo, small-sized graspable and hapticenabled robots. These robots operate on printed paper sheets and can be used as an interface for interacting with many virtual pointlike objects that reside on a plane [42]. Our design objective is to provide practical, easy to use and intuitive gamified upper-arm rehabilitation by using these novel tangible robots as game agents and objects (See Figure 1). By augmenting tangible objects commonly used for rehabilitation with robotic technologies such as precise localization and locomotion we hypothesize that various aspects of rehabilitation can be improved.

Designing games for rehabilitation requires input from multiple stakeholders. In order to meet design concerns of the end users, we iteratively designed a game by testing it in 4 different therapy centers and getting feedback from 7 therapists, 14 stroke patients, 2 patients with brachial plexus and 2 three-years-old children with hemiplegia. In this article, we present the iterative design of a rehabilitation game in collaboration with therapists, neurologists and patients to show the potential of an active tangible robot platform as a rehabilitation tool.

After discussing the related work in Section 2, we present the pilot tests and design phases co-developed with other stakeholders in Section 3. The initial promising results supporting our design rationales are shown in Section 4. Finally, we discuss our future study plans in Section 5 .

\section{RELATED WORK}

Recently, game-based rehabilitation approach gained popularity in upper arm functional recovery to increase patient motivation 
by integrating entertainment elements in the training tasks. Although most rehabilitation technologies have a common feature of providing motor practice in a compliant way, the type of technology used is diverse. This ranges from robotic arm based systems $[30,50,54]$ to tangible objects $[56,57]$ and commercial off-the-shelf game consoles $[5,16]$. Below, we provide a brief overview of these technologies.

Robot-assisted rehabilitation. Robot assisted rehabilitation can provide both accurate motion tracking and high volumes of repetitive movement training [28, 41]. Additionally they can provide solutions for severely impaired patients in both acute and chronic phases [14, 18, 30, 32, 35, 38]. The main strength of such systems is that it removes the need for a present therapist which can increase the accessibility to the therapy. However, the systems should be cost effective to be widely implemented [57]. In therapy use many therapists may stop using these devices, if set-up takes more than 5 minutes. Therefore, newly developed devices for physical training should be intuitive, easy and fast to set-up $[15,37]$.

Commercial virtual reality devices and video game consoles. Virtual reality combined with upper arm rehabilitation has promising results [10, 11, 23, 34, 45]. Advances in the commercial gaming device technology have led to a convergence of upper limb rehabilitation and commercial off-the-shelf video game consoles [2, 8, 41]. Systems such as the Nintendo Wii and Playstation II EyeToy deliver low cost virtual reality and report significant improvements in functional recovery of the participants $[5,13,16,25,44,46,47,60]$. Player interaction in a virtual environment is typically achieved by video capture, motion sensors, or a combination of both [41]. Motion tracking is mostly based on motion sensors such as Kinect and Wii Remote where the skeleton detection is fast. However, the use of these devices is often discussed to present an additional risk of seizures in the stroke population [29,49]. Nintendo has suggested that the risk of a seizure in healthy populations is 1 in $4000[39,41]$. Thomson et al. made a survey study with 112 therapists: Gaming was reported to be enjoyable but therapists described barriers related to time, space and cost; half of the therapists (51\%) reported at least one adverse event, such as fatigue, stiffness or pain [51]. Kumar et al. evaluated the usability of three lower-cost virtual reality rehabilitation games where P5 Glove and Kinect were used to record the movements [33]. People with stroke were found to desire motivating and easy-to-use games with clinical insights and encouragement from therapists. The results revealed the expectations of stroke survivors for games: the games should be improved by obtaining evidence for clinical effectiveness, including clinical feedback regarding improving functional abilities, adapting the games to the user's changing functional ability, and improving usability of the motion-tracking devices. It is claimed that lack of familiarity and steep learning curve of such devices may reduce the training efficiency among elderly users $[1,21]$.

Tangible interfaces. Recent researches explore tangible objects to provide motivating technology supported training systems in a residential environments by aiming accessible and affordable setups $[7,56]$. Wang et al. proposed a tangible gaming board with 64 RGB LED rotary encoders and initial results showed the acceptance of the system by stroke survivors [57]. Direct contact via touch interfaces is proposed to provide lower cognitive loads and a more suitable and intuitive alternative, especially for aging users [20].

Our proposal: Gamified rehabilitation with tangible robots. In our approach we aim to provide an intuitive, easy to use and easy to set up rehabilitation system and use tangible robots both as game agents and objects to be moved. The robot platform consists of low-cost, small-sized tangible mobile robots that operate on printed sheets of paper covered with a dot pattern that enables self-localization of the robot. The robots are designed to be simple to operate; all robots are connected wirelessly to a mobile device (a tablet or smartphone) that runs the activity and game logic. Each robot is capable of self-localization on the activity sheet; holonomic motion; 6 capacitive touch buttons (independently back-illuminated in full RGB) and wireless Bluetooth communication [42]. The platform provides fast and accurate localization of many robots that we can $\log$ in real time. The raw robot positions are also used, e.g. for onboard closed-loop motion control (including haptic feedback) and, on the external controller, for multi-robot formation control.

The activity itself is printed on paper sheets that can feature desired graphical game elements defined as active zones. These zones can be associated with specific robot behaviors to design game logic (e.g. blue wall zones activates assistive haptic behavior of the robot in Figure 2 and fruit zones represent target objects to be collected in our game). An activity is the combination of the paper elements, the robots with particular interaction modalities and the tablet that runs the activity software. As such, the role of the robots and paper depends on the design of each particular activity.

\section{ITERATIVE DESIGN OF THE REHABILITATION GAME}

The ultimate goal of this work is to obtain a game that is successful in motivating the patient throughout the rehabilitation process, which includes many stakeholders in addition to the patients such as neurologists, physiotherapists and occupational therapists. A traditional design process, where requirements are be gathered from all of these professionals as well as the patients and the final system is developed accordingly, would likely fail to achieve satisfactory results as the spectrum of stakeholders is too broad and diverse.

Instead, we opted to follow an iterative design process where various stakeholders were met with and the system tested in four different therapy centers/hospitals in four different cities of two different countries. Iteration 0,1 and 2 consisted mainly of design requirements meetings with and incremental software development of the game. Iteration 3 consisted of pilot experiments with adults and children where we tested our game for the first time with real, albeit healthy, users. Iterations 4 through 8 consist of experiments with different therapists and patients with varying degrees of impairment, and the implementation of improvements that resulted from these experiences. Below, we explain each iteration in detail.

\subsection{Iteration 0: Determining Design Rationales with the Neurologists}

In this initial iteration, design rationales of our game were determined with the input from neurologists. By considering the possible cognitive deterioration that may accompany physical impairments, 
the game elements and rules dare expected to be simple to play and easy to understand. According to the neurologists' experiences, the game should also include the features to change the level of difficulty according to the abilities of the patient besides simplicity. Therefore, the challenge level of the game should be configured for each patient with different levels of impairment.

\subsection{Iteration 1: Basic Game Elements and Rules for Simplicity}

With the previously obtained design rationales, we began discussing the nature of the game and its elements. We chose to implement a game inspired by Pacman to make the system possibly easier and more intuitive for the user to play with. The following game elements were designed with the input from physiotherapists:

- The Pacman robot: Includes both passive and active behaviors and is manually moved by the user.

- The Ghost robot(s): Chase(s) the Pacman in an autonomous way, the user does not manipulate them. The autonomous behavior is implemented to be robust against kidnapping to allow the therapist to move them to any other point on the map during the therapy.

- The paper: Seen in Figure 2 it consists of a Pacman-themed maze including walls, paths and fruits. The map size $(980 \times 420$ $\mathrm{mm}$ ) is designed to be suitable to the possible range of motions of the user's arm in sagittal and transverse planes while sitting on a table. Other visual items were added to the map to indicate the initial positioning of the Pacman and the Ghost(s).

- Game start: A simple multi-robot behavior is designed to initialize robot positions on the map in the beginning of the game which can help user to effectively start the game from the easiest position. When all robots reach their initial positions, Ghost(s) become(s) light-blue while Pacman becomes yellow and the Ghost(s) start(s) chasing.

- Rule 1: The user moves the Pacman along the paths without crashing into the walls and tries to collect 6 fruits on the map by bringing Pacman on top of the each fruit.

- Rule 2: If Ghost(s) catch(es) the Pacman, all collected fruits of the user are lost. We avoid using terms like death and life to prevent possible negative effects on the psychological status of the patients who are likely survivors of traumatic events.

- Game end: The game ends when all fruits are collected.

\subsection{Iteration 2: Game Elements for Difficulty Adaptation and Feedback}

With an initial game implementation, we began discussing the adjustment of difficulty for patients, which is challenging for those with upper limb impairments since the range of physical and cognitive deterioration is typically wide. In order to provide this adaptability, the main methods suggested by the neurologists were somehow manipulating the speed and the accuracy of the patient's motion. To achieve this, the following game elements were added which depends on some of the advantages of the robotic platform that we are using, such as haptic feedback and easy multiplication of programmable agents:
- Number of Ghosts: For a more challenging game the number of Ghosts chasing the Pacman can be changed. We hypothesize that increasing the number of Ghosts can increase the velocity of the user.

- Speed of the Ghost(s): The speed of the chasing Ghost(s) can be tuned to hypothetically increase or decrease the velocity of the user.

- Adaptive Ghost speed: During the game the Ghost speed can change dynamically, which adapts to the user's speed to make the game more challenging.

- Crash penalty: To force the user to be more accurate, crashing into the walls can be penalized by taking back the last collected fruit.

- Haptic disturbance: In order to increase the effort and attention of the patient, the neurologists hypothesized that patterned and random resistive control strategies can be used by applying haptic disturbance through the grasped Pacman. Several optional haptic features were thus implemented to investigate the effect of disturbative feedback on motor learning in future studies. Without haptic, the robot is like moving a mouse on the paper (robot is backdrivable). With haptic, the robot can apply up to $1 \mathrm{~N}$ force which is not enough to make user to do a motion involuntarily. If the user is passive and can not move his/her hand, he/she just puts force on the robot and the robot can not move. In this case the therapist assists patients to play. The haptic features are direction based vibrations and was taken as positive feature to increase perception of the motion by therapists, who did not anticipate any harmful movements.

- Assistive haptic stimulation: An assistive haptic stimulation control strategy was implemented to inform and assist the user when he/she crashes into a wall: Pacman does not intervene as long as the patient stays in between the walls; however, if the patient moves towards the walls the Pacman produces a restoring haptic force perpendicular to the wall. The intensity of the force is proportional to the deviation from the desired path. Some margin of deviation is tolerated before the restoring haptic force is provided.

- Game configuration for therapists: A configuration page was added to pre-game for therapists to decide difficulty level of the game by tuning all aforementioned features and game elements. This allows each therapist to configure the game from the tablet according to the type and state of the upper arm disability of the patient.

\subsection{Iteration 3: Mapping Rehabilitation Exercises to Game Activity Pool}

The crucial feature of a rehabilitation game is including the appropriate exercise motions. Naturally, since the base platform in our games is composed of tabletop robots, we focus on upper limb activities where the tangible robots serve as end-effectors for the patients' upper limbs. Although the robots perform movements in one plane (on the paper), joints of the limb holding the robot may still move in a three-dimensional space. Since the map is designed according to the possible range of motion of the human, following 

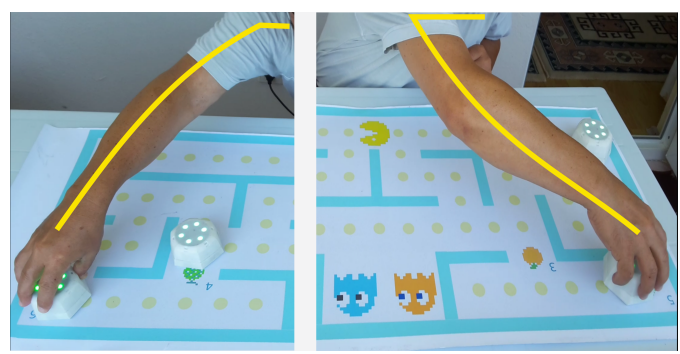

Figure 3: An example game scene of the user while performing shoulder horizontal abduction/adduction

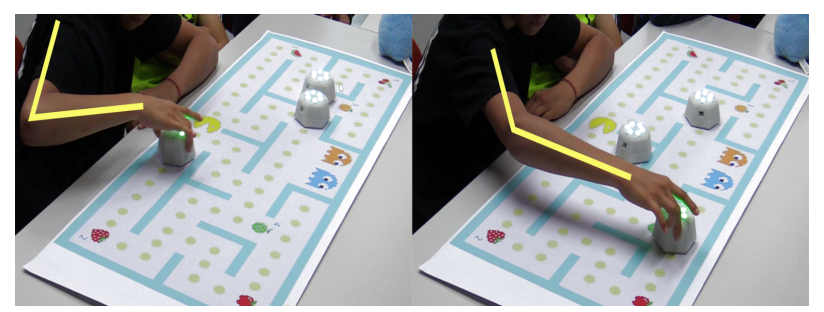

Figure 4: An example game scene of the user while performing elbow flexion/extension.
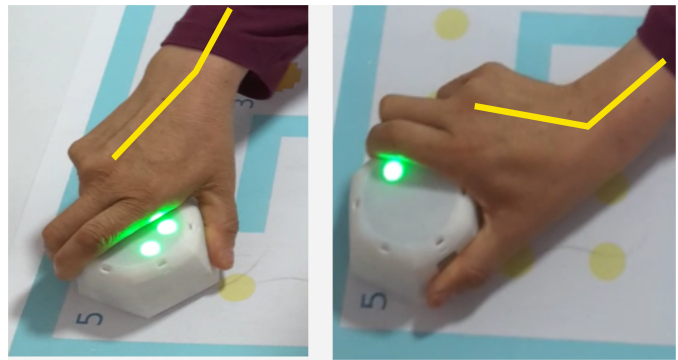

Figure 5: An example game scene of the user while performing wrist ulnar/radial deviation.

basic motions commonly used in stroke rehabilitation exercises are expected during the given corresponding actions:

- Moving the robot along the horizontal axis on the paper: shoulder abduction and adduction

- Moving the robot along the vertical axis on the paper: elbow flexion and extension

- Manipulating the robot: grasping and wrist ulnar and radial deviation

At this point, in order to make the game more challenging in terms of motion and increasing our motion pool, a rotating motion rule is added to the game. If this rule is enabled, the user should rotate the robot for a specific amount in order to be able to collect the fruits. Required angle for this optional turn motion is also determined by the therapist in the initial configuration settings $(45,90,135, \ldots, 360$ degrees).

We tested this motion pool and its mapping to our intended rehabilitation exercises with healthy adults and children. A total

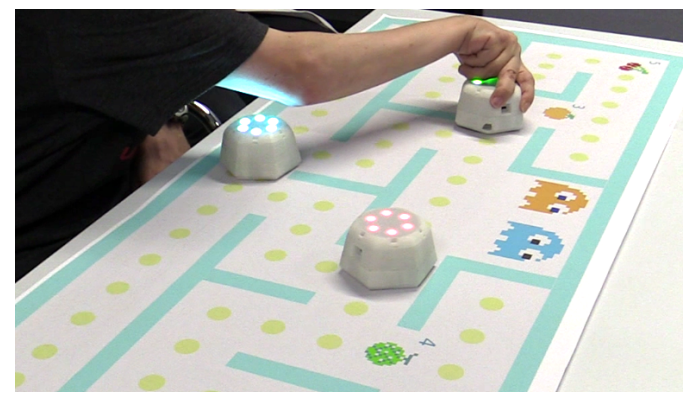

Figure 6: An example game scene of the patient while playing independently with 2 Ghost robots.

of 5 adults (27 to 57 years old) and 55 children (10 to 12 years old) played the game after a brief introduction with a given configuration that we desired to test at the time. In the case of children, the game time was limited to at most 3 to 4 minutes because of the availability of the participants. All of the expected rehabilitation motions listed-above were observed during game play (See Figures 3, 4 and 5). During iterative design process 7 therapists agreed that these movements would be useful in therapeutic contexts.

\subsection{Iteration 4: Severely Impaired Stroke Patient in Sion}

Beginning with this iteration, we began testing with our true end users, namely impaired patients that play the game with the help of and in the presence of neurologists and physiotherapists. A 68 years old female patient with 2 months stroke tested the game in a rehabilitation center located in Sion. She had severe upper limb impairment (right arm) and she used her healthy arm in 4 trials with different configurations of the game and then tried to move the robot with her impaired hand with the help of the neurologist.

Feedback and Changes: A demonstration session is added at the beginning of the experiment. A visual alert feedback using robot LEDs is added to inform the user when he/she crashes into the walls. Crash penalty and assistive haptic stimulation are made optional to adjust the path width for users who can move their hands with limited accuracy along the path; when these rules are not applied the path width does not mean anything for the game but just a visual to guide the user.

\subsection{Iteration 5: Moderately Impaired Stroke Patient in Sion}

Iteration 5 was done in the same center with a 49 year-old male patient with perinatal stroke (from birth). He had moderate hand and arm impairment (right arm) and played the game with his impaired arm 7 times with different configurations as seen in Figure 6.

Feedback and Changes: The patient enjoyed and gave positive feedback for the game and could play the game with incremental difficulty. After this test, a smaller maze $(620 \times 420 \mathrm{~mm})$ with smaller range of motion was designed for users with limited range of motion. A grasping aid to hold the hand on top of the robot is suggested by the neurologist to help patients play the game more easily. 

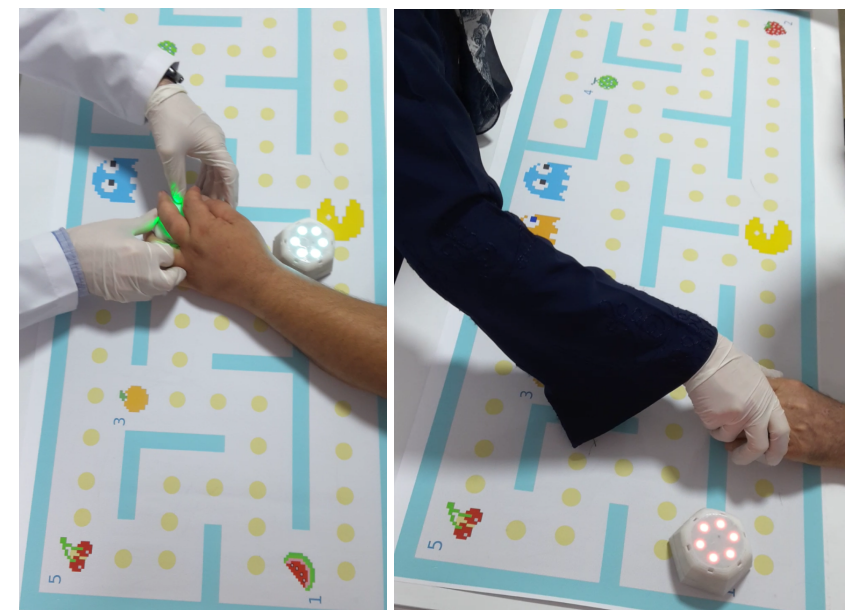

Figure 7: Example game scenes of the patients assisted by their therapists.

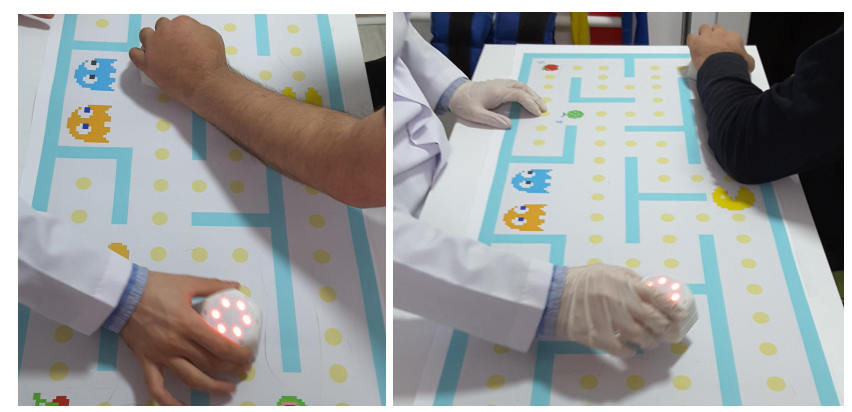

Figure 8: Tangibility and kidnap robustness allow the therapist to intervene easily.
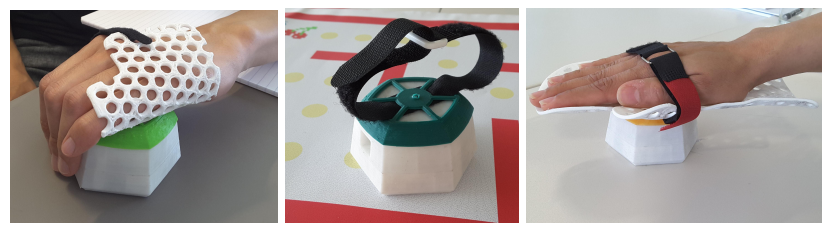

Figure 9: Grasping aids assisting patient to hold the robot.

\subsection{Iteration 6: 5 Stroke Patients, 2 Brachial Plexus Patients and 3 Therapists in Istanbul}

5 stroke patients with varying degree of impairment played the game to test the usability and playability in a rehabilitation center located in Istanbul. 1 female patient and 1 male patient were able to play the game without any help with changing difficulty levels for 30 minutes each. 3 male patients played the game with the assistance of their therapists as seen Figure 7. 3 therapists tested the game and the possible grasping aid design were also discussed with them. Therapists also suggested to use the game with children with brachial plexus. Afterwards, children with brachial plexus tested the game.
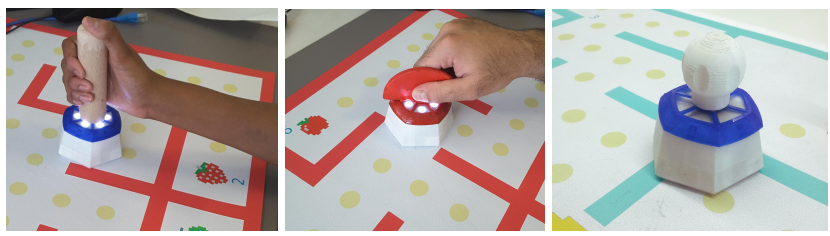

Figure 10: Grasp and grip tools.

Feedback and Changes: The patients enjoyed the game and therapists gave positive feedback about tangibility and kidnap robustness of the game. Interventions by the therapist where he/she moved the chasing Ghost(s) anywhere were frequently used (see Figure 8) to tangibly adjust the dynamic of the game. After these tests, new grasping aids that can be attached on top of the robot were designed. In order to allow for training of different grasps, different knobs attachable to the robot were suggested. This modularity is suggested to make the system adaptable to a specific condition or to perform additional exercises like cylindrical grasp, spherical grasp or disk grip. Designed grasping aids and attachments can be seen in Figure 9 and 10.

\subsection{Iteration 7: Occupational Therapy with Grasping Aids in Geneva}

Two occupational therapy sessions with conventional therapy methods were observed in Geneva. During the therapies, the game was played by 1 male patient and 1 female patient with stroke, who tested some of the handlers. Two grasping aids were observed to help the female user to hold the robot during the game, and one knob is found useful for the male patient, who was able to hold robot without any other help.

\subsection{Iteration 8: Stroke and Hemiplegia from age 3 to 77 in Lausanne}

This iteration featured more contribution from therapists who wanted to discover the attitudes of smaller age groups towards the game. Two 3 years old children with hemiplegia played the game with the small map and even though they have attention deficits one played the game for 25 minutes the other played for 35 minutes, which was an exceedingly promising result for the therapists. Naturally, a possible novelty effect should be taken into account and further tests should be done. Additionally, therapists wanted the children to move the robot with both arms to increase coordination, which was a new motion type for our system. Finally, even though both children understood the rule of escaping from the Ghost, both preferred to catch the Ghosts which is a behavior we should take into account in further design.

Another child (age 11) with stroke played the game for $45 \mathrm{~min}$ utes. After he mentioned his problem of opening bottles, the therapist suggested to use the game with different attachments in rotation enabled mode as a way of training for opening bottles in daily life. The therapist mentioned at this point that they do not prefer to use commercial off-the-shelf video game consoles with kids for the reason that children tend to move their torso instead of moving the arms in full range (which is not a desired motion in that therapy) 
since they are standing up. This anecdotal feedback hints at the usefulness of tabletop systems.

Finally, our system was used with elderly stroke patients. 3 patients played the game independently and one patient played with the help of her therapist. In this instance, the physiotherapist explicitly stated the reason of not frequently using other technologies (e.g Kinect, robotic arms) as (1) The time to prepare the system and (2) The difficulty to intervene to the interaction during the game or task. The fact that we set up our game in a regular therapy room in a few minutes on a small table and did not need for the patient to move to another room was acclaimed by this physiotherapist. Additionally, during the game play the therapist appreciated that she could intervene to the game and hold the patient's hand whenever she wanted to correct the motion, and stated that this joint tangibility provides a better way of conveying the therapy.

\section{RESULTS}

During the iteration process mostly the therapists or the patient determined the configuration of the game according to the abilities of the user. We logged the motion and game data of the patients then analyzed the data to see the relationship between designed game elements and performance of the user.

In data processing we only used data of the users that can play the game independently during the above mentioned iterative phases (54 trials of 11 participants) to see the effect of game elements to only patients' performances. We excluded the data of patients played with the assistance of the therapists. Following performance and accuracy measures are decided to be used:

- Distance: Quantity of the motion (in $\mathrm{mm}$ ) which is calculated by the $\mathrm{x}$ and $\mathrm{y}$ position of Pacman robot logged in every $100 \mathrm{~ms}$.

- Time: Total time spent between the start and end of the game (which means successfully collecting all the fruits).

- Mean velocity of the user: The mean of the speed of the user calculated from the position.

- Deviance mean: The mean of the amount of deviance from the middle of the path measured in $\mathrm{mm}$.

- Crash events: Total number of crashings into a wall during the game.

These evaluation metrics are selected by the neurologists and therapists. In design phase, we focused on possible game mechanics (active agents, haptics, and challenges) that can be implemented with any game using Cellulo platform. The metrics we used to evaluate performance were linked to game elements such as challenges (wall crashing and turning), timing, quality and quantity of the motion (deviance and total motion).

We conducted various one way ANOVA tests without assuming equal variances across conditions, with the number of Ghost robots ( 1 or 2 ) as the independent variable and the different performance and accuracy measures as dependent variable and observed the following:

(1) the distance travelled by the user against one active agent (Ghost robot) is significantly less than that the user against two active agents (Ghost robots) $(\mathrm{F}[1,21.76]=6.18, \mathrm{p}=.02$, on the left in Figure 11);

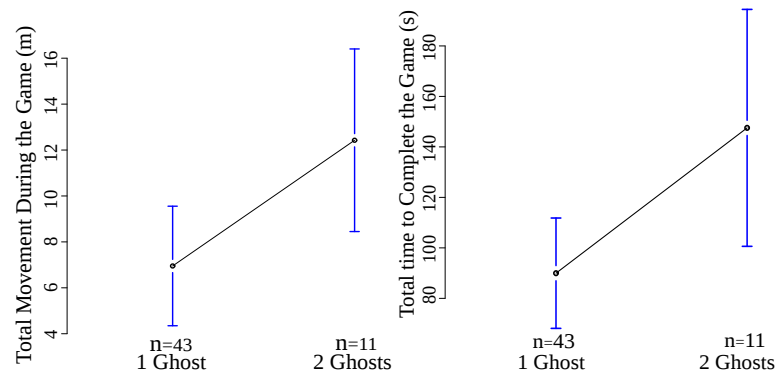

Figure 11: Distance travelled by Pacman and time taken to finish a game for the different number of Ghost robots. The blue bars represent the $95 \%$ confidence intervals.
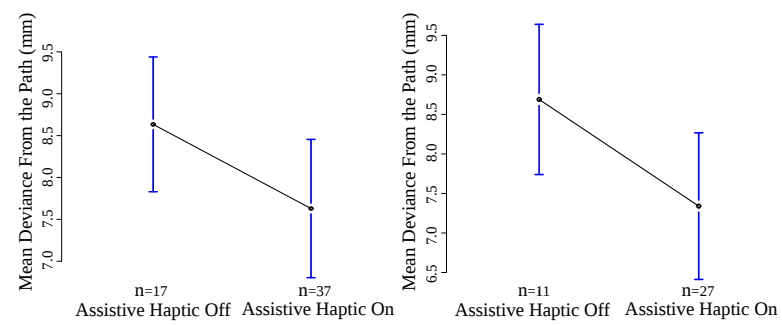

Figure 12: Mean deviance from the path for the assistive haptic stimulation on and off, for all the patients and for only the stroke patients. The blue bars represent the $95 \%$ confidence intervals.

(2) the total time taken to finish the game against one Ghost robot is significantly less than that against two Ghost robots $(\mathrm{F}[1,15.72]=5.91, \mathrm{p}=.02$, on the right in Figure 11$)$

(3) the deviance against one Ghost robot is significantly less than that against two Ghost robots $(\mathrm{F}[1,16.68]=5.45, \mathrm{p}=$ $.03)$;

Next, we conducted the similar one way ANOVA tests using the presence (or absence) of the assistive haptic stimulation as a feedback mechanism when the user crashes a wall as an independent variable, and we observed that the deviance with the assistive haptic is marginally less than the deviance without the assistive haptic $(F[1,46.57]=3.26, p=.07$, on the left in Figure 12). This difference is significant for the stroke patients $(F[1,30.31]=4.72, p$ $=.03$, on the right in Figure 12).

Further, we observed a significantly positive correlation $(\mathrm{r}(50)$ $=0.35, \mathrm{p}=.01$ ) between total distance travelled by Pacman and speed of the Ghost robot(s). Also we observed another significantly positive correlation $(\mathrm{r}(50)=0.63, \mathrm{p}<.0001)$ between the velocity of the Pacman and speed of the Ghost robot(s).

Moreover, another one way ANOVA with the crash wall penalty on (or off) as an independent variable showed that the deviance from the path was significantly less in when the wall crash penalty was on as compared to the cases when the wall crash penalty was off $(F[1,17.55]=4.17, \mathrm{p}=.05$, Figure 13).

We did not see any order effect in between the trials. However, we observed a tendency of increased velocity over time (See Figure 14) which might be a signal of learning which should be explored 


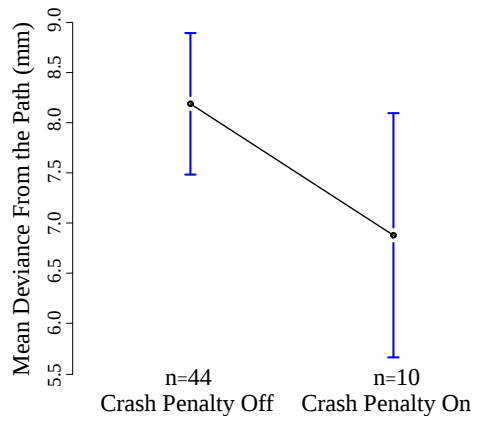

Figure 13: Mean deviance from the path for the crash wall penalty on and off. The blue bars represent the $95 \%$ confidence intervals.
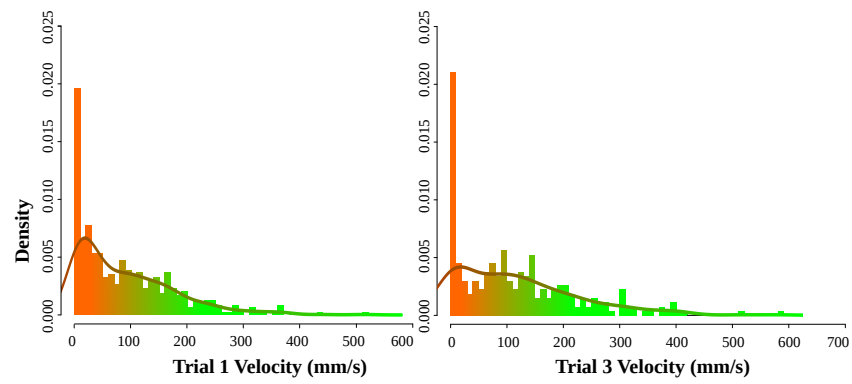

Figure 14: The effect of trial number on velocity density.

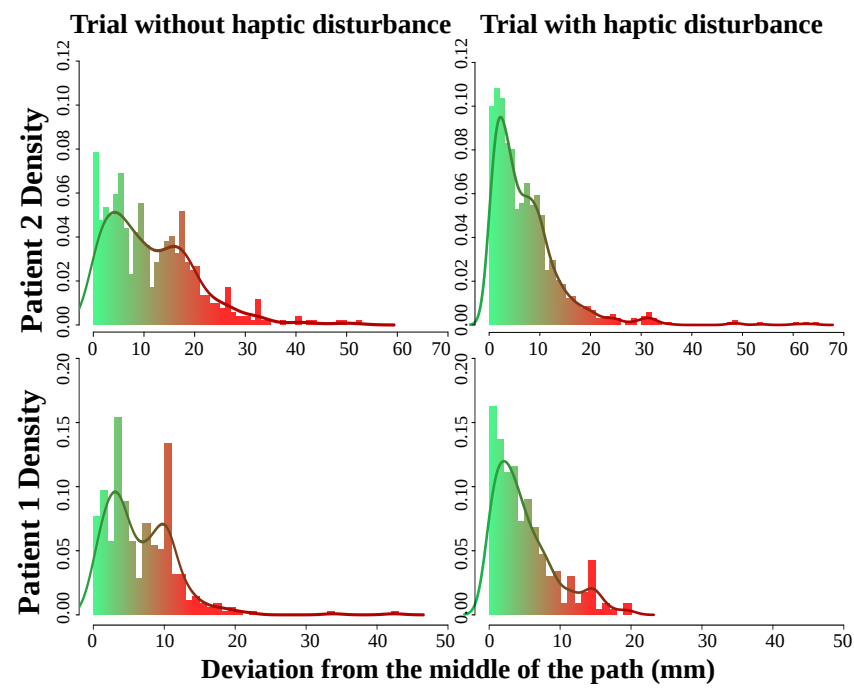

Figure 15: The effect of haptic disturbance on the deviance from the middle path density.

deeply in the future studies. Haptic disturbance was added to a couple of trials and it was not enough to do a statistical test. But when we compare the deviance values of trials with and without haptic disturbance, the density curve is more skewed to left when the haptic is on as seen in Figure 15. This may imply that when we introduce the disturbance, accuracy of the motion increases. These tendencies cannot yet be accepted as robust scientific evidence and the effectiveness of haptic disturbance approach should be tested in more controlled experiments with concerning the possible order effect and the effect of other game elements.

In the light of these preliminary results, in therapy if the focus is to have more repetitions, one can increase the number of agents or increase the chasing speed of the agents which result in more motion. If the focus is accurate functionality of the upper limb such as precise motion along a path, one can use games that have haptic assistive feedback, games with less active agents or games with crash wall penalty rule. On the other hand if the focus is faster motion of the limb, one can use games with agents having higher chasing speed.

\section{CONCLUSION AND FUTURE WORK}

In this study we described our iterative design process, which is involving the diverse stakeholder groups, for an upper extremity rehabilitation game with a novel tangible robot platform. We tested our design rationales and results showed that with our game elements, such as assistive haptic stimulation, wall crash penalty and increased number of ghosts, we are able to affect the accuracy of the user's motion; whereas with increasing the number of active robots in the game we can affect the time spent on the game and the amount of the user's motion. These results may help us to understand the relationship between performance metrics of the user and game elements such as number of active agents, speed of active agents and haptic assistance that can be integrated to any game. Understanding this relationship may assist us to design adaptive games with difficulty adjustment for different users who has different level of impairment and/or different therapy focus. Although the final design is endorsed by the neurologists and physiotherapists, in order to show the impact of the platform we will conduct long term rehabilitation studies. We are planning to find out the learning effect through time and show the versatility of the game by focusing on rehabilitation of different health problems such as hemiparesis, hemispatial neglect, attention deficit and poor visual discrimination. With this platform we also aim to provide social rehabilitation as well as physical. Recent studies found out that motivational properties of the game depends on the personal preference for competition or cooperation of the patient $[26,40,43]$. Therefore, the game should include collaborative two-player, competitive two-player and single player options in the future. Integration of two player games into the system brings with the need of performance-based adaptive control strategies. We are aiming to monitor the performance of both the patient and the second player then adapt various aspects of assistance according to the performance of the users.

\section{ACKNOWLEDGMENTS}

This work has been partially supported by the Swiss National Science Foundation through the National Centre of Competence in Research Robotics and also partially supported by the Defitech Foundation (Morges, Switzerland). The authors would like to thank the therapists at the Service de Neuropsychologie et de Neuroréhabilitation at the Vaud University Hospital Center (CHUV), and all 
the other therapists and patients for their valuable contributions to our design process.

\section{REFERENCES}

[1] R. Aarhus, E. Grönvall, S. B. Larsen, and S. Wollsen. Turning training into play: Embodied gaming, seniors, physical training and motivation. Gerontechnology, 10(2):110-120, 2011

[2] G. Alankus, A. Lazar, M. May, and C. Kelleher. Towards customizable games for stroke rehabilitation. In Proceedings of the SIGCHI Conference on Human Factors in Computing Systems, pages 2113-2122. ACM, 2010.

[3] T. Apted, J. Kay, and A. Quigley. Tabletop sharing of digital photographs for the elderly. In Proceedings of the SIGCHI conference on Human Factors in computing systems, pages 781-790. ACM, 2006.

[4] L. Axelrod, G. Fitzpatrick, J. Burridge, S. Mawson, P. Smith, T. Rodden, and I. Ricketts. The reality of homes fit for heroes: design challenges for rehabilitation technology at home. fournal of Assistive Technologies, 3(2):35-43, 2009.

[5] B. Bonnechère, B. Jansen, L. Omelina, J. Van Sint, et al. The use of commer cial video games in rehabilitation: a systematic review. International journal of rehabilitation research, 39(4):277-290, 2016.

[6] B. Bonnechère, B. Jansen, L. Omelina, and S. Van Sint Jan. Do patients perform their exercises at home and why (not)? a survey on patients' habits during rehabilitation exercises. Ulutas Med f, 2:41-6, 2016.

[7] J. W. Burke, M. McNeill, D. Charles, P. J. Morrow, J. Crosbie, and S. McDonough Augmented reality games for upper-limb stroke rehabilitation. In Games and Virtual Worlds for Serious Applications (VS-GAMES), 2010 Second International Conference on, pages 75-78. IEEE, 2010.

[8] J. W. Burke, M. McNeill, D. K. Charles, P. J. Morrow, J. H. Crosbie, and S. M. McDonough. Optimising engagement for stroke rehabilitation using serious games. The Visual Computer, 25(12):1085, 2009.

[9] M. S. Cameirao, I. B. S. Bermúdez, E. Duarte Oller, and P. F. Verschure. The rehabilitation gaming system: a review. Stud Health Technol Inform, 145(6), 2009

[10] M. S. Cameirão, S. B. i Badia, E. D. Oller, and P. F. Verschure. Neurorehabilitation using the virtual reality based rehabilitation gaming system: methodology, design, psychometrics, usability and validation. Fournal of neuroengineering and rehabilitation, 7(1):48, 2010.

[11] M. S. Cameirão, S. B. i Badia, L. Zimmerli, E. D. Oller, and P. F. Verschure. The rehabilitation gaming system: a virtual reality based system for the evaluation and rehabilitation of motor deficits. In Virtual Rehabilitation, 2007, pages 29-33. IEEE, 2007.

[12] J. H. Cauraugh and J. J. Summers. Neural plasticity and bilateral movements: a rehabilitation approach for chronic stroke. Progress in neurobiology, 75(5):309-320, 2005.

[13] J. Crosbie, S. Lennon, M. McGoldrick, M. McNeill, and S. McDonough. Virtual reality in the rehabilitation of the arm after hemiplegic stroke: a randomized controlled pilot study. Clinical Rehabilitation, 26(9):798-806, 2012.

[14] J. J. Daly, N. Hogan, E. M. Perepezko, H. I. Krebs, et al. Response to upper-limb robotics and functional neuromuscular stimulation following stroke. Fournal of rehabilitation research and development, 42(6):723, 2005.

[15] M. P. Dijkers, R. Erlandson, K. Kristy, D. Geer, A. Nichols, et al. Patient and staff acceptance of robotic technology in occupational therapy: a pilot study. fournal of rehabilitation research and development, 28(2):33, 1991.

[16] L. R. A. Dos Santos, A. A. Carregosa, M. R. Masruha, P. A. Dos Santos, M. L. D. S. Coêlho, D. D. Ferraz, and N. M. D. S. Ribeiro. The use of nintendo wii in the rehabilitation of poststroke patients: a systematic review. fournal of Stroke and Cerebrovascular Diseases, 24(10):2298-2305, 2015.

[17] S. E. Fasoli, H. I. Krebs, J. Stein, W. R. Frontera, and N. Hogan. Effects of robotic therapy on motor impairment and recovery in chronic stroke. Archives of physical medicine and rehabilitation, 84(4):477-482, 2003.

[18] S. E. Fasoli, H. I. Krebs, J. Stein, W. R. Frontera, R. Hughes, and N. Hogan. Robotic therapy for chronic motor impairments after stroke: Follow-up results. Archives of physical medicine and rehabilitation, 85(7):1106-1111, 2004.

[19] G. Fitzpatrick, M. Balaam, and S. R. Egglestone. Involving stroke survivors in designing for rehabilitation at home. Therapeutic Strategies A Challenge for User Involvement in Design, page 13, 2010.

[20] F. Garcia-Sanjuan, J. Jaen, and V. Nacher. Tangibot: a tangible-mediated robot to support cognitive games for ageing peopleâĂTa usability study. Pervasive and Mobile Computing, 34:91-105, 2017.

[21] K. M. Gerling, F. P. Schulte, and M. Masuch. Designing and evaluating digital games for frail elderly persons. In Proceedings of the 8th international conference on advances in computer entertainment technology, page 62. ACM, 2011.

[22] C. Grefkes and N. S. Ward. Cortical reorganization after stroke: how much and how functional? The Neuroscientist, 20(1):56-70, 2014.

[23] M. K. Holden. Virtual environments for motor rehabilitation. Cyberpsychology \& behavior, 8(3):187-211, 2005.

[24] J. A. Hosp and A. R. Luft. Cortical plasticity during motor learning and recovery after ischemic stroke. Neural plasticity, 2011, 2011.
[25] J. K. Hsu, R. Thibodeau, S. J. Wong, D. Zukiwsky, S. Cecile, and D. M. Walton. A 'wii' bit of fun: The effects of adding nintendo wii ${ }^{\circledR}$ bowling to a standard exercise regimen for residents of long-term care with upper extremity dysfunction. Physiotherapy Theory and Practice, 27(3):185-193, 2011.

[26] M. Hudson. Applying exergaming input to standard commercial digital games. In Proceedings of the 2016 CHI Conference Extended Abstracts on Human Factors in Computing Systems, pages 1886-1895. ACM, 2016.

[27] Y.-X. Hung, P.-C. Huang, K.-T. Chen, and W.-C. Chu. What do stroke patients look for in game-based rehabilitation: a survey study. Medicine, 95(11), 2016.

[28] M. J. Johnson. Recent trends in robot-assisted therapy environments to improve real-life functional performance after stroke. fournal of NeuroEngineering and Rehabilitation, 3(1):29, 2006.

[29] L. Y. Joo, T. S. Yin, D. Xu, E. Thia, P. F. Chia, C. W. K. Kuah, and K. K. He. A feasibility study using interactive commercial off-the-shelf computer gaming in upper limb rehabilitation in patients after stroke. Journal of rehabilitation medicine, 42(5):437-441, 2010.

[30] H. Kim, L. M. Miller, I. Fedulow, M. Simkins, G. M. Abrams, N. Byl, and J. Rosen. Kinematic data analysis for post-stroke patients following bilateral versus unilateral rehabilitation with an upper limb wearable robotic system. IEEE Transactions on Neural Systems and Rehabilitation Engineering, 21(2):153-164, 2013.

[31] H. I. Krebs, S. Mernoff, S. E. Fasoli, R. Hughes, J. Stein, and N. Hogan. A comparison of functional and impairment-based robotic training in severe to moderate chronic stroke: a pilot study. NeuroRehabilitation, 23(1):81-87, 2008

[32] H. I. Krebs, B. T. Volpe, M. Ferraro, S. Fasoli, J. Palazzolo, B. Rohrer, L. Edelstein, and N. Hogan. Robot-aided neurorehabilitation: from evidence-based to sciencebased rehabilitation. Topics in stroke rehabilitation, 8(4):54-70, 2002.

[33] J. A. Kumar, M. Binal Motawar PT, and K. Lakshminarayanan. Usability evaluation of low-cost virtual reality hand and arm rehabilitation games. fournal of rehabilitation research and development, 53(3):321, 2016.

[34] K. E. Laver, S. George, S. Thomas, J. E. Deutsch, and M. Crotty. Virtual reality for stroke rehabilitation. The Cochrane Library, 2015.

[35] A. C. Lo, P. D. Guarino, L. G. Richards, J. K. Haselkorn, G. F. Wittenberg, D. G. Federman, R. J. Ringer, T. H. Wagner, H. I. Krebs, B. T. Volpe, et al. Robot-assisted therapy for long-term upper-limb impairment after stroke. New England fournal of Medicine, 362(19):1772-1783, 2010.

[36] M. Ma and K. Bechkoum. Serious games for movement therapy after stroke. In Systems, Man and Cybernetics, 2008. SMC 2008. IEEE International Conference on, pages 1872-1877. IEEE, 2008.

[37] P. Maciejasz, J. Eschweiler, K. Gerlach-Hahn, A. Jansen-Troy, and S. Leonhardt. A survey on robotic devices for upper limb rehabilitation. fournal of neuroengineering and rehabilitation, 11(1):3, 2014

[38] T. Nef, G. Quinter, R. Müller, and R. Riener. Effects of arm training with the robotic device armin i in chronic stroke: three single cases. Neurodegenerative diseases, 6(5-6):240-251, 2009.

[39] Nintendo. Wii health and safety precautions.

[40] D. Novak, A. Nagle, U. Keller, and R. Riener. Increasing motivation in robotaided arm rehabilitation with competitive and cooperative gameplay. Fournal of neuroengineering and rehabilitation, 11(1):64, 2014.

[41] O. ONeil, C. Gatzidis, and I. Swain. A state of the art survey in the use of video games for upper limb stroke rehabilitation. In Virtual, Augmented Reality and Serious Games for Healthcare 1, pages 345-370. Springer, 2014.

[42] A. Özgür, W. Johal, F. Mondada, and P. Dillenbourg. Haptic-enabled handheld mobile robots: Design and analysis. In Proceedings of the 2017 CHI Conference on Human Factors in Computing Systems, pages 2449-2461. ACM, 2017.

[43] T. Park, C. Yoo, S. P. Choe, B. Park, and J. Song. Transforming solitary exercises into social exergames. In Proceedings of the ACM 2012 conference on Computer Supported Cooperative Work, pages 863-866. ACM, 2012.

[44] D. Rand, R. Kizony, and P. T. L. Weiss. The sony playstation ii eyetoy: low-cost virtual reality for use in rehabilitation. Journal of neurologic physical therapy, 32(4):155-163, 2008

[45] R. Sanchez, D. Reinkensmeyer, P. Shah, J. Liu, S. Rao, R. Smith, S. Cramer, T. Rahman, and J. Bobrow. Monitoring functional arm movement for home-based therapy after stroke. In Engineering in Medicine and Biology Society, 2004. IEMBS'04. 26th Annual International Conference of the IEEE, volume 2, pages 4787-4790. IEEE, 2004.

[46] G. Saposnik, M. Levin, S. O. R. C. S. W. Group, et al. Virtual reality in stroke rehabilitation. Stroke, 42(5):1380-1386, 2011.

[47] G. Saposnik, R. Teasell, M. Mamdani, J. Hall, W. McIlroy, D. Cheung, K. E. Thorpe, L. G. Cohen, M. Bayley, et al. Effectiveness of virtual reality using wii gaming technology in stroke rehabilitation. Stroke, 41(7):1477-1484, 2010

[48] M. Shaughnessy, B. M. Resnick, and R. F. Macko. Testing a model of post-stroke exercise behavior. Rehabilitation nursing, 31(1):15-21, 2006.

[49] M. M. Shoja, R. S. Tubbs, A. Malekian, A. H. J. Rouhi, M. Barzgar, and W. J. Oakes. Video game epilepsy in the twentieth century: a review. Child's Nervous System, 23(3):265-267, 2007

[50] P. Staubli, T. Nef, V. Klamroth-Marganska, and R. Riener. Effects of intensive arm training with the rehabilitation robot armin ii in chronic stroke patients: four single-cases. Journal of neuroengineering and rehabilitation, 6(1):46, 2009. 
[51] K. Thomson, A. Pollock, C. Bugge, and M. Brady. Commercial gaming devices for stroke upper limb rehabilitation: a systematic review. International fournal of Stroke, 9(4):479-488, 2014

[52] K. Thomson, A. Pollock, C. Bugge, and M. C. Brady. Commercial gaming devices for stroke upper limb rehabilitation: a survey of current practice. Disability and Rehabilitation: Assistive Technology, 11(6):454-461, 2016.

[53] B. Ullmer and H. Ishii. Emerging frameworks for tangible user interfaces. IBM systems journal, 39(3.4):915-931, 2000.

[54] A. Van Delden, C. L. E. Peper, G. Kwakkel, and P. J. Beek. A systematic review of bilateral upper limb training devices for poststroke rehabilitation. Stroke research and treatment, 2012, 2012.

[55] J. H. Van der Lee, R. C. Wagenaar, G. J. Lankhorst, T. W. Vogelaar, W. L. Devillé, and L. M. Bouter. Forced use of the upper extremity in chronic stroke patients. Stroke, 30(11):2369-2375, 1999.

[56] M. Vandermaesen, T. De Weyer, K. Luyten, and K. Coninx. Physicube: providing tangible interaction in a pervasive upper-limb rehabilitation system. In Proceedings of the 8th International Conference on Tangible, Embedded and Embodied
Interaction, pages 85-92. ACM, 2014

[57] P. Wang, G. C. H. Koh, C. G. Boucharenc, T. M. Xu, C. C. Yen, et al. Developing a tangible gaming board for post-stroke upper limb functional training. In Proceedings of the Tenth International Conference on Tangible, Embedded, and Embodied Interaction, pages 617-624. ACM, 2017.

[58] J. Whitall, S. M. Waller, K. H. Silver, and R. F. Macko. Repetitive bilateral arm training with rhythmic auditory cueing improves motor function in chronic hemiparetic stroke. Stroke, 31(10):2390-2395, 2000.

[59] H. Woldag and H. Hummelsheim. Evidence-based physiotherapeutic concepts for improving arm and hand function in stroke patients: a review. fournal of neurology, 249(5), 2002.

[60] G. Yavuzer, A. Senel, M. Atay, and H. Stam. "playstation eyetoy games"improve upper extremity-related motor functioning in subacute stroke: a randomized controlled clinical trial. European journal of physical and rehabilitation medicine, 44(3):237-244, 2008 